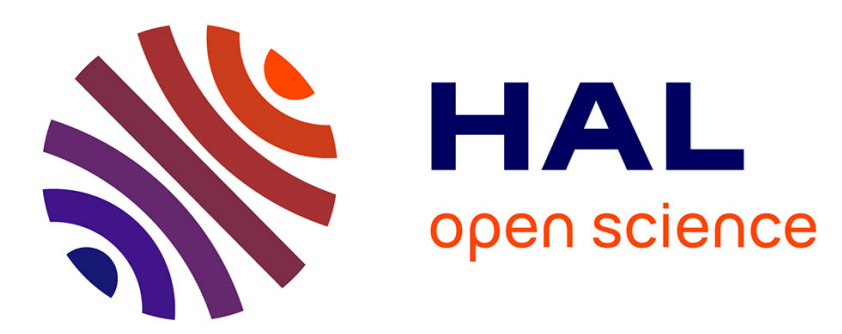

\title{
Oral rinse MMP-8 point-of-care test identifies patients with strong periodontal inflammatory burden
}

Jussi Leppilahti, Minna-Maija Ahonen, Marcela Hernández, Sarvan Munjal, Lutz Netuschil, Veli-Jukka Uitto, Timo Sorsa, Päivi Mäntylä

\section{- To cite this version:}

Jussi Leppilahti, Minna-Maija Ahonen, Marcela Hernández, Sarvan Munjal, Lutz Netuschil, et al.. Oral rinse MMP-8 point-of-care test identifies patients with strong periodontal inflammatory burden. Oral Diseases, 2010, 17 (1), pp.115. 10.1111/j.1601-0825.2010.01716.x . hal-00599812

\section{HAL Id: hal-00599812 \\ https://hal.science/hal-00599812}

Submitted on 11 Jun 2011

HAL is a multi-disciplinary open access archive for the deposit and dissemination of scientific research documents, whether they are published or not. The documents may come from teaching and research institutions in France or abroad, or from public or private research centers.
L'archive ouverte pluridisciplinaire HAL, est destinée au dépôt et à la diffusion de documents scientifiques de niveau recherche, publiés ou non, émanant des établissements d'enseignement et de recherche français ou étrangers, des laboratoires publics ou privés. 


\section{ORALIDISEASES}

\section{Oral rinse MMP-8 point-of-care test identifies patients with strong periodontal inflammatory burden}

\begin{tabular}{|r|l|}
\hline Journal: & Oral Diseases \\
\hline Manuscript ID: & ODI-02-10-OM-1554.R1 \\
\hline Manuscript Type: & Original Manuscript \\
\hline Date Submitted by the & $12-$ Mar-2010 \\
\hline Author: & $\begin{array}{r}\text { Leppilahti, Jussi; University of Helsinki, Institute of Dentistry } \\
\text { Ahonen, Minna-Maija; University of Helsinki, Institute of Dentistry } \\
\text { Munalnal, Sarvan; dentognostics GmbH } \\
\text { Netuschil, Lutz; dentognostics GmbH } \\
\text { Uitto, Veli-Jukka; University of Helsinki, Institute of Dentistry; } \\
\text { Helsinki University Central Hospital, Department of Oral and } \\
\text { Maxillofacial Diseases } \\
\text { Sorsa, Timo; University of Helsinki, Institute of Dentistry; Helsinki } \\
\text { University Hospital, Department of Oral and Maxillofacial Diseases } \\
\text { Mäntylä, Päivi; University of Helsinki, Institute of Dentistry }\end{array}$ \\
\hline Keywords: & $\begin{array}{l}\text { oral rinse, biomarker, chair-side test, periodontal diagnostics, MMP- } \\
8\end{array}$ \\
\hline
\end{tabular}

\section{S) ScholaroNE \\ Manuscript Central}


Oral rinse MMP-8 point-of-care test identifies patients with strong periodontal inflammatory $\underline{\text { burden }}$

Leppilahti Jussi $\mathbf{M}^{1}$, Ahonen Minna-Maija ${ }^{1}$, Hernández Marcela ${ }^{3}$, Munjal Sarvan ${ }^{4}$, Netuschil Lutz ${ }^{4}$, Uitto Veli-Jukka ${ }^{1,2}$, Sorsa Timo ${ }^{1,2}$, Mäntylä Päivi ${ }^{1}$

${ }^{1}$ Institute of Dentistry, University of Helsinki, Helsinki, Finland

${ }^{2}$ Department of Oral and Maxillofacial Diseases, Helsinki University Central Hospital, Helsinki, Finland

${ }^{3}$ Periodontal Biology Laboratory, Faculty of Dentistry, University of Chile, Santiago, Chile

${ }^{4}$ dentognostics GmbH, Jena, Germany

Running title: Oral rinse MMP-8 testing

Key words: Oral rinse, biomarker, chair-side test, periodontal diagnostics, MMP-8

\section{Correspondence should be addressed to:}

Dr Päivi Mäntylä, DDS, PhD

Institute of Dentistry, University of Helsinki

P.O.Box 41

FIN-00014 University of Helsinki

Finland

Tel: +358919127217

Fax: +358919127519

E-mail: paivi.mantyla@helsinki.fi 


\section{Abstract}

Objective: The aim of the study was to determine whether oral rinse MMP- 8 levels, measured by three different methods, TIMP-1 levels and elastase activity differentiate subjects with different periodontal condition; and second, to find out if MMP-8 levels were comparable among the methods used.

Methods: MMP-8 levels were analyzed with an immunofluorometric method (IFMA), dentoAnalyzer and commercial ELISA. Also TIMP-1 levels and elastase activity were measured. For statistical analysis 214 study subjects were categorized into four groups, specified by the presence and number of moderate $(4-5 \mathrm{~mm})$ and deep $(\geq 6 \mathrm{~mm})$ periodontal pockets, and bleeding on probing percentage.

Results: MMP-8 levels especially measured by dentoAnalyzer and adjusted to the number of teeth per subject differentiated the study group with strong periodontal inflammatory burden from groups with lower levels. This was also verified with receiver operating characteristic (ROC) analysis. Elastase activity associated with higher IFMA and dentoAnalyzer MMP-8 levels. IFMA MMP-8/TIMP and dentoAnalyzer MMP-8/TIMP-1 tended to be higher with the increasing level of periodontal inflammatory burden. TIMP-1 levels decreased with increasing age.

Conclusions: Oral rinse MMP-8 together with TIMP-1 analysis may have potential in complementary periodontal diagnostics. dentoAnalyzer can be applied in quantitative oral rinse chair side biomarker diagnostics. 


\section{Introduction}

Periodontitis is a very common inflammatory disease in humans. Though bacteria are the initiating factor, periodontitis is not a classical infectious disease. Majority of the causative organisms are also present in the healthy mouth (Socransky \& Haffajee 2005), and the host response modified by environmental and behavioural factors plays a key role in disease development, progression and in the maintenance of the treatment result. Despite the etiology or type of periodontitis, the products of host response and tissue destruction are similar. Markers of inflammatory process from periodontal tissues migrate into the periodontal pockets within a serum originating fluid, gingival crevicular fluid (GCF) (Uitto et al., 2003), and further from gingival crevices/pockets into oral cavity where GCF associates with saliva. The more inflamed the periodontal tissues and the deeper the periodontal pockets are, the more GCF is excreted and the more it contains inflammatory markers (Uitto et al., 2003). This also means that the inflammatory burden of periodontium may be reflected in saliva (Uitto et al., 1990; Sorsa et al., 1990).

Unlike in medicine, biochemical testing in dentistry has not yet been taken into everyday clinical practice despite the advantages in supplementation of traditional diagnostics. GCF has been used for site specific diagnostic studies of periodontal disease status, and diagnostic tests for host derived markers have been developed (Uitto et al., 2003; Sorsa et al., 2004). Inflammatory markers from GCF analysed individually or in combination may be valuable in the identification of individual sites or teeth at risk of periodontitis progression. Saliva can also be regarded as a diagnostic fluid but unlike GCF, it mirrors more generally the oral status and is more unspecific. Whole saliva contains sloughed epithelial cells from oral mucous membranes, nasopharyngeal discharge, food debris, bacteria and their products and, in dentate subjects, its content is also affected by GCF flow from gingival pockets. An oral rinse sample can be regarded as a fluid containing mainly the GCF from all periodontal pockets (Sorsa et al., 1990; Uitto et al., 1990, 2003). Thus, analysis of oral rinse may be useful in the diagnosis of general periodontal disease status, early identification of subjects at risk of periodontitis (Uitto et al., 2003), evaluation of periodontal treatment results or the need of hygiene visits during maintenance and in the follow up of the effect of modifying medication over host response (for example low-dose doxycycline, LDD) (Reinhardt et al., 2010). 
Matrix metalloproteinases (MMPs) and their inhibitors and polymorphonuclear leukocyte (PMN) elastase could be candidates as diagnostic markers in oral rinse specimens (Uitto et al., 2003; Sorsa et al., 2004). Among MMPs, especially GCF MMP-8 levels have been found to be increased in periodontitis (Mäntylä et al., 2003, 2006). The deeper the periodontal pockets are, the higher are the levels of MMP-8 at individual sites, and the levels decrease by periodontal hygiene phase treatment. GCF MMP-8 levels remain low in treated and stable periodontitis while in subjects with tendency to progression of periodontitis or poor treatment response (refractory periodontitis) GCF indicates repeatedly high MMP-8 levels. Though smoking may decrease GCF MMP-8 levels, there may also be smoking subjects who repeatedly have especially high GCF MMP-8 levels and poor treatment response (Mäntylä et al., 2006).

Salivary MMP-8 levels have been suggested serving as a biomarker of periodontitis and applicable as a point-of-care test because MMP-8 levels in saliva have been found to correlate with periodontal disease severity (Miller et al., 2006; Ramseier et al., 2009). Herr et al. (2007) have introduced a microfluidic assay for point-of-care rapid quantitation of biomarkers from human saliva which in validation with MMP-8 identified the periodontitis subjects.

Also salivary elastase-like activity has been concluded to reflect the severity of periodontal destruction because elevated activity levels have been found in untreated periodontitis subjects' saliva, which decrease after periodontitis hygiene phase treatment (Nieminen et al., 1993). The results were similar in the oral water-rinse samples: a significant correlation was noticed between oral rinse elastase activity and the average of community index of periodontal treatment needs (CPITN) (Uitto et al., 1996).

In our previous study (Sorsa et al., 2010) we analyzed GCF MMP-8 levels by different methods and found that methods which based on the same monoclonal antibody against MMP-8 [immunofluorometric method (IFMA), MMP-8 specific chair-side dip-stick test and dentoAnalyzer appliance] gave comparable results. In this current paper we have used IFMA, dentoAnalyzer as well as commercially available Amersham ELISA assay to determine MMP-8 levels in oral rinse samples from subjects with different periodontal health status expressed as different levels of periodontal inflammatory burden. Additionally, TIMP-1 levels and detection of elastase-like activity were screened in oral rinse samples by Amersham ELISA and a test utilising a chromogenic substrate (Nieminen et al., 1993; Uitto et al., 1996), respectively. The aim was to find out if these markers analyzed with the applied methods individually or in combination, have diagnostic 
potential in differentiation of subjects with different periodontal inflammatory burden level. In addition we wished to find out whether the results of MMP-8 analysis obtained by used methods were comparable.

\section{Materials and Methods}

\section{Patients}

In this cross-sectional study 214 adult patients with age range 44-78 years (mean \pm sd $\underline{57.2 \pm 7.9)}$ were randomly selected from dental public health clinics in cities of Helsinki and Vantaa. Patients in need of antibiotic prophylaxis or suffering of a contagious disease were excluded. The protocol was clearly explained to all study subjects and institutional review board-approved informed consents were signed. The study protocol was approved by the ethical committee of the Institute of Dentistry, University of Helsinki and Helsinki University Central Hospital, and the experiments were undertaken with the understanding and written consent of each subject and according to ethical principles, including the World Medical Association Declaration of Helsinki.

All study subjects were examined clinically: bleeding on probing (BOP) was registered dichotomously on four tooth surfaces and pocket probing depths (PPD) by Florida-probe device (Florida Probe Corporation, USA) on six tooth surfaces. PPD was recorded if $\geq 4 \mathrm{~mm}$. Examinations were done by two calibrated general dentists. Background characteristics were recorded by using questionnaires. Oral rinse samples were taken from all patients.

Patients were categorized into four groups according to their periodontal inflammatory burden, determined by combining the Periodontal Inflammatory Burden Index (PIBI) described by Lindy et al. (2008) and BOP\%. PIBI is calculated by adding the number of periodontal sites indicating moderate periodontitis (number of periodontal pockets with PPD 4-5 $\mathrm{mm}=\mathrm{Nmod}$ ) to the weighted number of periodontal sites indicating advanced periodontitis (number of periodontal pockets with $P P D \geq 6 \mathrm{~mm}$ multiplied by two $=\mathrm{Nadv}$ ).

Periodontal inflammatory markers are conducted into oral rinse within the GCF. Flow of GCF is increased in inflamed tissues and is also proportional to the area of inflamed epithelial surface of the periodontal sulcus/pocket (Armitage, 1995; Weidlich et al., 2001). BOP\% indicates inflammation in the periodontal tissues and the PIBI reflects periodontal inflammatory injury (Lindy et al., 2008). Based on this we reasoned to integrate PIBI and BOP\% into one index (PIBI $\mathrm{x}$ 
BOP\%) with the purpose to indicate the GCF flow. We hypothesized that the higher the flow of GCF, the higher is the level of inflammatory markers in oral rinses, and that it reflects the periodontal health status.

According to the categorization methods described above we formed four groups of study subjects: (1) 36 subjects were regarded as periodontally healthy (BOP $<10 \%$ of teeth surfaces and no deepened periodontal pockets; (2) 21 subjects with $\mathrm{BOP} \geq 10 \%$ of teeth surfaces but no deepened periodontal pockets, mild periodontal inflammatory burden, regarded clinically as gingivitis. Patients diagnosed to have deepened periodontal pockets (PPD $\geq 4 \mathrm{~mm}$ ) were categorized into two groups according the PIBI x BOP \%: 104 subjects had PIBI x BOP \% 1-100 (group 3; moderate periodontal inflammatory burden) and 53 patients had PIBI x BOP $\% \geq 100$ (group 4; strong periodontal inflammatory burden). Threshold of 100 was chosen to discriminate moderate inflammatory burden (clinically regarded as minor or early periodontitis) from strong inflammatory burden (clinically more advanced disease).

\section{Oral rinse samples}

Oral rinse samples were taken as follows: $1 \mathrm{ml}$ of tap water was placed into the subject's oral cavity with a disposable plastic pipette. Subjects rinsed their mouth with tap water for 1 minute and the rinse was collected into collection tubes (Nieminen et al., 1993; Uitto et al., 1996). On the same day an aliquot of the specimen was taken for elastase activity testing and the rest of the sample was frozen on the same day for further MMP-8 and TIMP-1 analyses, (Uitto et al., 1996).

\section{MMP-8, TIMP-1 and elastase determinations}

All samples were analyzed for MMP-8 levels by three different immunological assays: dentoAnalyzer device (Munjal et al., 2007; Sorsa et al., 2010), a time-resolved immunofluorometric assay (Hanemaaijer et al., 1997; Mäntylä et al., 2003, 2006) and a commercial ELISA kit by Amersham (Munjal et al., 2007; Sorsa et al., 2010). Briefly, dentoAnalyzer is designed as a portable user-friendly bench-top instrument. The device applies a sandwich based immunoassay technology using ABICAP filters. TIMP-1 levels were measured with Amersham ELISA (Tuomainen et al., 2007; Rautelin et al., 2009). Amersham ELISA analyses of MMP-8 and TIMP-1 levels were done according to manufacturer's instructions. MMP-8 and TIMP-1 results $(\mathrm{ng} / \mathrm{ml})$ were expressed as levels per subject and per total number of teeth/subject.

Deleted: B
Deleted: B
Deleted: B

Deleted: At

Deleted: next

Deleted: tests 
Elastase activity test was done based on the method described by Nieminen et al. (1993) and Uitto et al. (1996). The chromogenic substrate succinyl-alanyl-valine-p-nitroanlilide (Sigma Co, St Louis, MO, USA) was dissolved in a solution with the following final concentrations: $1 \mathrm{mM}$ substrate, $0.1 \%$ dimethyl sulfoxide, $0.2 \mathrm{M} \mathrm{NaCl}$ and $0.05 \mathrm{M}$ Tris- $\mathrm{HCl}$ buffer, $\mathrm{pH}$ 7.5. The enzyme activity is expressed as optical density after subtracting the $0 \mathrm{~h}$ value. Optical densities were measured at nominal/categorical scale by comparing the colour of test plates to a coloured slide designed for eye comparison of optical densities. Optical densities were analyzed by two observers to minimize chance of misinterpretation. Optical densities were categorized according to previously published results, which proved good correlation between elastase activity and the number of deep periodontal pockets (Uitto et al., 1996). 0.2 OD was used as a cut off level for a significant positive elastase activity and levels below the threshold of 0.2 OD were regarded as negative (Uitto et al., 1996).

\section{Data analysis}

Non-parametric Kruskal-Wallis or Mann-Whitney U test for independent samples was used for testing statistical significances of differences between study groups and bivariate non-linear Spearman correlation, for testing of correlations between results obtained by different MMP-8 detection methods. An association of nominal variables in crosstables was tested by Cramér V test. The diagnostic accuracy of MMP-8 measuring methods used was evaluated using Receiver Operating Charasteristics (ROC) curve analysis. p-values $<0.05$ were considered to be statistically significant. Statistical analyses and tests were done by SPSS-software (version 17.0).

\section{Results}

\section{Clinical parameters and demographic data}

Subject groups, clinical parameters and demographic characteristics are shown in Table 1. Association between consensus risk factors (Genco, 1996) for chronic periodontitis; gender, tobacco smoking and diabetes, and different periodontal indecces were tested (Table 2). In general terms, male gender and tobacco smoking were associated with higher values of all periodontal indecess used in the study: PIBI, BOP\%, and PIBI x BOP\% ( $1<0.001)$, except for the association between male gender and $\mathrm{BOP} \%$, that was insignificant $(\mathrm{p}=0.323)$. However, diabetes associated with 
decreased $\mathrm{BOP} \%(\mathrm{p}=0.016)$ in this study population (Table 2). Diabetic subjects were not examined as a separate group, and they could belong to any of the study groups 1-4.

\section{Assocation between MMP-8 and periodontal inflammatory burden}

MMP-8 levels increased along with periodontal inflammatatory burden (Table 3). Enzyme levels measured by dentoAnalyzer were significantly different among groups $(p=0.018)$ and differences were more pronounced when MMP-8 levels were adjusted to the number of teeth for both, dentoAnalyzer (Figure 1) and IFMA ( $\mathrm{p}=0.007$ and $\mathrm{p}=0.032$, respectively).

\section{Correlations between MMP-8 detection methods}

Correlation between MMP-8 levels measured with IFMA and dentoAnalyzer were tested with Spearman correlation test which gave a correlation coefficient 0.691 (statistically significant at $\mathrm{p}<$ 0.01 level). No correlation was found between MMP-8 analysis by dentoAnalyzer or IFMA and Amersham ELISA. MMP-8 levels measured by Amersham ELISA test did not correlate with the values of periodontal parameters.

\section{Statistical outliers}

Based on statistical outliers of MMP-8 levels adjusted to the number of teeth (Figure 1), we determined the threshold for peak levels of MMP-8/number of teeth measured by both dentoAnalyzer and IFMA. The thresholds were determined at $15 \mathrm{ng} / \mathrm{ml}$ level for dentoAnalyzer results corresponding $80 \mathrm{ng} / \mathrm{ml}$ levels for IFMA. Percentages of MMP-8/number of teeth peak levels in each study group are shown in Table 4. The higher the severity of periodontal disease (heavier periodontal inflammatory burden) was, the higher the percentage of peak values of MMP8/number of teeth, except for group 3 (Table 4). The difference was statistically significant when MMP-8 levels were measured by dentoAnalyzer $(p=0.016$; Table 4$)$. The Amersham ELISA MMP-8 levels did not express statistical outliers.

MMP-8/TIMP-1 ratio and TIMP-1 levels

MMP-8/TIMP-1 molar ratios increased together with periodontal inflammatory burden when measured by dentoAnalyzer $(\mathrm{p}=0.033$, Table 3$)$.

TIMP-1 levels decreased progressively with inflammatory burden and with age group, but 
only the latter differences were significant $(\mathrm{p}<0.001$, Figure 2$)$.

\section{Elastase activity}

A statistically significant difference $(\mathrm{p}<0.001)$ in MMP-8/number of teeth levels between groups with negative and positive elastase activity test results was detected when MMP-8 was measured by dentoAnalyzer or IFMA but not when measured by Amersham ELISA. The difference of dentoAnalyzer MMP-8/number of teeth median values between elastase activity groups was also tested separately in each patient group and confirmed to be statistically significant (for groups with mild, moderate and strong inflammatory burden, i.e. groups 2,3 , and $4 \mathrm{p}<0.001$; for periodontally

Deleted: group 1 healthy, i.e. group $1 \mathrm{p}=0.029$; Figure 3). dentoAnalyzer MMP-8/number of teeth levels increased in line with study groups (inflammatory burden), except for group 3 with moderate inflammatory burden, as mentioned earlier in the Results and seen in Figure 1. However, the increase was especially pronounced when the study groups were further categorized according to the elastase activity (positive elastase activities: $\mathrm{p}=0.002$; negative elastase activity $\mathrm{p}=0.322 \mathrm{~ns}$; Figure 3 ).

\section{ROC-curve analysis}

A Receiver Operating Charasteristic (ROC) analysis was ran to evaluate the diagnostic sensitivity and specificity of dentoAnalyzer MMP-8/number of teeth. The ability of dentoAnalyzer MMP-8/number of teeth to distinguish between periodontally healthy subjects and subjects with different levels of periodontal inflammatory burden (groups 2, 3 and 4 with mild, moderate and

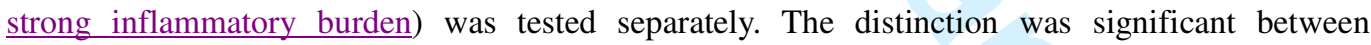
periodontally healthy (group 1) and subjects with strong periodontal inflammatory burden (group 4) (Figure 4). The area under the curve was 0.701 ( $p=0.002 ; 95 \%$ confidence interval $0.59-0.81$ ). 


\section{Discussion}

In this study the subjects were not selected based on a defined periodontal disease status. Instead, the randomly selected study subjects were categorised based on the level of periodontal inflammatory burden expressed as numbers and depths of deepened periodontal pockets and percentage of bleeding on probing.

MMP-8 levels in oral rinse samples were higher in subjects with the strongest periodontal inflammatory burden than in subjects with less inflammatory changes when oral rinse levels were adjusted to number of teeth, especially when measured with dentoAnalyzer appliance. This finding was confirmed with ROC analysis. Similarly, MMP-8/TIMP-1 ratios increased in subjects with strong periodontal inflammatory burden when MMP-8 was measured by dentoAnalyzer. MMP-8 Deleted: rose Deleted: highest increments were especially pronounced in subjects showing detectable elastase activity.

The measurement of host originating oral biomarker levels in periodontal diagnostics has faced the problem that it has been extremely difficult to define the "normal" levels of various biomarkers. Biomarkers which are expressed at high levels in periodontal inflammation can be detected at lower levels also in periodontally healthy oral cavity, obviously as an indication of the physiologic turnover of oral tissues (McCulloch, 1994, Uitto et al., 2003). Besides, the individual expression of biomarkers in healthy state as well as in disease may vary because of genetic variation (McCulloch, 1994; Uitto et al., 2003).

MMP-8 can be regarded as a central enzyme in periodontitis development and progression (Sorsa et al., 2004, 2006). It may be useful in site specific diagnostics to find out if individual sites or teeth are at risk of progression of periodontitis when analysed from GCF. Mäntylä et al. (2006) found that repeatedly high MMP-8 levels in GCF tested with a MMP-8 specific chair side dip-stick test identified sites/teeth which were at risk of progression of periodontal attachment loss. Site specific MMP-8 testing also identified the subjects with poor response to conventional periodontal treatment, i.e. scaling and root planing. These subjects had multiple sites with an unstable character, and these sites were repeatedly test positive during the 12 month maintenance phase of periodontal treatment. Repeated testing may thus give more reliable diagnostic information than single testing in site specific biomarker diagnostics.

Smoking has been found in several studies to decrease MMP-8 levels in GCF because of the effects on local blood circulation and lowered signs of inflammation (Söder, 1999; Söder et al., 
2002). However, Mäntylä et al. (2006) detected the highest MMP-8 GCF levels in smoking subjects with poor response to conventional periodontal treatment (scaling and root planing, SRP). Thus it cannot be regarded categorically that all smoking periodontitis patients have lower levels of MMP-8 in GCF and further in oral rinse sample.

Periodontitis is regarded to be cyclic by nature, and part of the dentition may be in active and progressing phase of periodontitis while other teeth may be quiescent or even in the phase of recovery (Carranza \& Camargo 2006 ). Cyclic nature of periodontitis may be even more challenging for oral rinse than for GCF based diagnostics. In an oral rinse sample the GCF is pooled from all periodontal pockets, and the collected oral rinse sample represents GCFs from whole periodontium. For this reason the definition of the cut off level for the biomarker which would be indicative for the deterioration of periodontal health is demanding. For this reason an oral rinse sample biomarker analysis can be used only for a rough assessment of the periodontal inflammatory level. The activity of periodontitis can only be established by monitoring patients longitudinally, and the progression can only be assessed site specifically. In our current study we did not address the question of disease activity or progression. Instead, we wanted to find out, if the surface area for subgingival biofilm accumulation expressed as periodontal pocketing and the simultaneous bleeding on probing, which points out the possibility of an ongoing inflammation, are reflected in an oral rinse sample.

In this current study some subjects with clinically healthy periodontium (no deepened periodontal pockets and bleeding on probing $<10 \%$ ) as well as some subjects with mild to moderate inflammatory burden with limited numbers of deepened periodontal pockets indicating gingivitis or mild periodontitis, expressed high levels of oral rinse MMP-8 which was detected by IFMA and dentoAnalyzer. High oral rinse MMP-8 levels in subjects with no clinical periodontal disease and in subjects with mild to moderate periodontal inflammatory burden were statistical outliers or peak values and were expressed only by some individuals in groups of subjects with healthy periodontium or with mild or moderate inflammatory burden. The cut off level for "positive" MMP-8 finding in an oral rinse sample measured by dentoAnalyzer and adjusted to the number of teeth which indicates a person at risk, may be the level above which the statistical outliers or peak values in groups of subjects with mild to moderate periodontal inflammatory burden are situated in box and whiskers plots, i.e. $15 \mathrm{ng} / \mathrm{ml}$. These peak values in periodontally healthy subjects' samples may indicate a proinflammatory condition in subjects which may have an

\begin{tabular}{|l|}
\hline Deleted: of \\
\hline Deleted: 2 and 3 ( \\
\hline Deleted: ) \\
\hline Deleted: (group 1) \\
\hline
\end{tabular}


aggressive host response and can be susceptible to develop periodontitis, i.e. subjects with a high "normal" MMP-8 level. In subjects with mild to strong inflammatory burden, oral fluid MMP-8 at least partly originates from inflamed marginal gingival tissues but levels above the risk level (15 $\mathrm{ng} / \mathrm{ml}$ ) may also indicate the subjects who are at risk to progress from gingivitis to periodontitis, or at risk of progression of periodontitis.

MMP-8 levels measured by IFMA and dentoAnalyzer, but not with Amersham ELISA, were comparable. The obvious reason for the high correlation between IFMA and dentoAnalyzer MMP-8 levels is the same monoclonal antibody shared by both methods (Hanemaaijer et al., 1997; Mäntylä et al., 2003, 2006; Sorsa et al., 2010), but based on its association with the severity of periodontal inflammation, this antibody may also be applicable and promising in oral fluid periodontal diagnostics. The antibody used in dentoAnalyzer and IFMA exert high sensitivity to target active forms of MMP-8 (Sorsa et al., 2010; Hanemaaijer et al., 1997), whereas the ELISA might test proform and thus target total MMP-8. IFMA is a method which can be used only in research laboratories and for this reason it is not ideal for rapid chair-side use in a dentist's office (Sorsa et al., 2010). dentoAnalyzer has been designed for chair-side use and it gives numeric quantitative information about the analysis in 15 minutes (Munjal et al., 2007; Sorsa et al., 2010).

It has been suggested that the simultaneous measurement of several oral fluid biomarkers could be beneficial in periodontal diagnostics. In this current study oral rinse MMP-8 levels and elastase activity, both enzymes which originate especially from PMN cells, associated with the level of periodontal inflammatory burden. However, it is not necessarily useful to measure many biomarkers with the same cellular origin. In this study we also analysed TIMP-1 levels, which is a tissue inhibitor of MMPs, in oral rinse samples. MMP-8/TIMP-1 ratios had a trend to be higher in the group of subjects with strong inflammatory burden and the ratio was significantly higher when MMP-8 levels were analysed by dentoAnalyzer.

A novel finding was that oral rinse TIMP-1 levels decrease with increasing age which can be regarded as an indication of age related changes in tissue homeostasis. Age as such has not been regarded as a risk factor for periodontitis. In aging skin, expression of TIMP-1 is decreased with fibroblast senescence (Hornebeck, 2003). Aging may have a similar effect in oral mucous membranes and periodontal tissues. Relevant age related changes related to TIMP-1 decrease can also be supported by the findings of Benatti et al. (2008) indicating that aging modulates important biological properties of periodontal ligament cells and favours extracellular matrix remodelling. 
Diagnostic tests are always somewhat unreliable and they should only be supplementary when the likelihood of a disease is estimated (Pauletto et al., 2000; Uitto et al., 2003; Sorsa et al., 2006). Very seldom a single biochemical test is diagnostically fully reliable but it may be helpful as an adjunct to clinical examination (Mäntylä et al., 2003, 2006). No positive test finding in clinic should lead to treatment unless clinical findings are in line, and vice versa, the negative finding shouldn't lead to the neglect of health promoting measures if clinical findings indicate inflammation. Based on our findings, the oral rinse sample analysis of MMP- 8 could be clinically useful in rough screening to indentify individuals who are at risk to develop periodontitis or in analysing the individual level of host response. Simultaneous analysis of MMP-8 and TIMP-1 could be beneficial. Oral rinse sample analysis may be useful in defining the optimal period between periodontal maintenance visits after active periodontal treatment. During the active treatment phase it would be possible to monitor the decrease of oral rinse MMP-8 levels, and oral rinse MMP-8 levels at the end of the active treatment phase would show the individual level to keep the host response in control. In this regard, the testing could give valuable information about the control of inflammation which today is based on the clinical findings, i.e. shallower or eliminated periodontal pockets and less bleeding on probing. At best the biomarker level could be monitored by a home test to indicate when the cut off of for possibly unsafe biomarker level is reached. Also the effect of MMP-8 inhibiting low-dose doxycycline (LDD) medication could be monitored by analysing the oral rinse MMP-8 levels to find out when a possible brake in medication would be possible or when the medication should be taken again (Golub et al., 2008; Reinhardt et al., 2010).

In conclusion, MMP-8 testing of oral rinse samples may be beneficial in periodontal diagnostics, but should be applied at this phase of research with caution. Testing may be adjunctive to other clinical screening methods if it is used correctly. Today it cannot be ruled out that there may be false negative findings because of inadequate knowledge about the safe cut off levels, and the levels may vary between different individuals depending on individual biomarker profiles. Simultaneous analysis of TIMP-1 may be beneficial. 


\section{Acknowledgements}

This study was supported by grants from the Academy of Finland, the Research Foundation of Helsinki University Central Hospital, the Finnish Dental Society Apollonia, and the Fondecyt Foundation of Chile. Timo Sorsa is an inventor of US-patents 5652227, 5736341, 5866432 and 6143476. dentognostics $\mathrm{GmbH}$ has been the scientific developer of the dentoAnalyzer. However, dentognostics $\mathrm{GmbH}$ does not manufacture, sell or distribute this device. 


\section{References}

Armitage GC (1995). Clinical evaluation of periodontal diseases. Periodontol 2000. 7: 39-53.

Benatti BB, Silvério KG, Casati MZ, Sallum EA, Nociti FH Jr (2008). Influence of aging on biological properties of periodontal ligament cells. Connect Tissue Res. 49: 401-408.

Genco RJ (1996). Current view of risk factors for periodontal diseases. J Periodontol. 67: 10411049.

Golub LM, Lee HM, Stoner JA, Sorsa T, Reinhardt RA, Wolff MS, Ryan ME, Nummikoski PV, Payne JB (2008). Subantimicrobial-dose doxycycline modulates gingival crevicular fluid biomarkers of periodontitis in postmenopausal osteopenic women. J Periodontol. 79: 1409-1418.

Hanemaaijer R, Sorsa T, Konttinen YT, Ding Y, Sutinen M, Visser H, van Hinsbergh VW, Helaakoski T, Kainulainen T, Rönkä H, Tschesche H, Salo T (1997). T. Matrix metalloproteinase-8 is expressed in rheumatoid synovial fibroblasts and endothelial cells. Regulation by tumor necrosis factor-alpha and doxycycline. J Biol Chem. 272: 31504-31509.

Herr AE, Hatch AV, Throckmorton DJ, Tran HM, Brennan JS, Giannobile WV, Singh AK (2007). Microfluidic immunoassays as rapid saliva-based clinical diagnostics. Proc Natl Acad Sci U S A. 104: $5268-5273$.

Hornebeck W (2003). Down-regulation of tissue inhibitor of matrix metalloprotease-1 (TIMP-1) in aged human skin contributes to matrix degradation and impaired cell growth and survival. Pathol Biol. 51: 569-573.

Lindy O, Suomalainen K, Mäkelä M, Lindy S (2008). Statin use is associated with fewer periodontal lesions: A retrospective study. BMC Oral Health. 15; 8: 16.

Carranza FA, Camargo PM (2006). The periodontal pocket. In: Newman MG, Takei HH, Klokkevold PR, Carranza FA, eds., Carranza's clinical periodontotology. 10th Edition. Saunders Elsevier: Louisville, USA, pp.434-451. 
McCulloch CA (1994). Host enzymes in gingival crevicular fluid as diagnostic indicators of periodontitis. J Clin Periodontol. 21: 497-506.

Miller CS, King CP Jr, Langub MC, Kryscio RJ, Thomas MV (2006). Salivary biomarkers of existing periodontal disease: a cross-sectional study. J Am Dent Assoc. 137: 322-329.

Munjal S, Miethe P, Netuschil L, Struck F, Maier K, Bauermeister C (2007). Immunoassay-based diagnostic point-of-care technology for oral specimen. Ann NY Acad Sci. 1098: 486-489.

Mäntylä P, Stenman M, Kinane DF, Tikanoja S, Luoto H, Salo T, Sorsa T (2003). Gingival crevicular fluid collagenase-2 (MMP-8) test stick for chair-side monitoring of periodontitis. $J$ Periodontal Res. 38:436-439.

Mäntylä P, Stenman M, Kinane D, Salo T, Suomalainen K, Tikanoja S, Sorsa T (2006). Monitoring periodontal disease status in smokers and nonsmokers using a gingival crevicular fluid matrix metalloproteinase-8-specific chair-side test. J Periodontal Res. 41: 503-512.

$$
\mid
$$

Nieminen A, Nordlund L, Uitto VJ (1993). The effect of treatment on the activity of salivary proteases and glycosidases in adults with advanced periodontitis. J Periodontol. 64: 297-301.

Pauletto NC, Liede K, Nieminen A, Larjava H, Uitto VJ (2000). Effect of cigarette smoking on oral elastase activity in adult periodontitis patients. J Periodontol. 71: 58-62.

Ramseier CA, Kinney JS, Herr AE, Braun T, Sugai JV, Shelburne CA, Rayburn LA, Tran HM, Singh AK, Giannobile WV (2009). Identification of pathogen and host-response markers correlated with periodontal disease. J Periodontol. 80: 436-446.

Rautelin HI, Oksanen AM, Veijola LI, Sipponen PI, Tervahartiala TI, Sorsa TA, Lauhio A (2009). Enhanced systemic matrix metalloproteinase response in Helicobacter pylori gastritis. Ann Med. 41: $208-215$.

Reinhardt RA, Stoner JA, Golub LM, Lee HM, Nummikoski PV, Sorsa T, Payne JB (2010). Association of gingival crevicular fluid biomarkers during periodontal maintenance with 
subsequent progressive periodontitis. J Periodontol. in press 2010.

Socransky SS, Haffajee AD (2005). Periodontal microbial ecology. Periodontol 2000. 38: 135-187.

Sorsa T, Suomalainen K, Uitto VJ (1990). The role of gingival crevicular fluid and salivary interstitial collagenases in human periodontal diseases. Arch Oral Biol. 35 Suppl: 193S-196S.

Sorsa T, Tjäderhane L, Salo T (2004). Matrix metalloproteinases (MMPs) in oral diseases. Oral Dis. 10: $311-318$.

Sorsa T, Tjäderhane L, Konttinen YT, Lauhio A, Salo T, Lee HM, Golub LM, Brown DL, Mäntylä P (2006). Matrix metalloproteinases: contribution to pathogenesis, diagnosis and treatment of periodontal inflammation. Ann Med. 38: 306-321.

Sorsa T, Hernández M, Leppilahti J, Munjal S, Lutz Netuschil L, Mäntylä P (2010). Detection of gingival crevicular fluid MMP-8 levels with different laboratory and chair-side methods. Oral Dis. 16: $39-45$

Söder B (1999). Neutrophil elastase activity, levels of prostaglandin E2, and matrix metalloproteinase- 8 in refractory periodontitis in smokers and non-smokers. Acta Odontol Scand. 57: $77-82$.

Söder B, Jin LJ, Wickholm S (2002). Granulocyte elastase, matrix metalloproteinase-8 and prostaglandin E2 in gingival crevicular fluid in matched clinical sites in smokers and non-smokers with persistent periodontitis. J Clin Periodontol. 29: 384-391.

Tuomainen AM, Nyyssönen K, Laukkanen JA, Tervahartiala T, Tuomainen TP, Salonen JT, Sorsa T, Pussinen PJ (2007). Serum matrix metalloproteinase-8 concentrations are associated with cardiovascular outcome in men. Arterioscler Thromb Vasc Biol. 27: 2722-2728.

Uitto VJ, Suomalainen K, Sorsa T (1990). Salivary collagenase. Origin, characteristics and relationship to periodontal health. J Periodontal Res. 25: 135-142.

Uitto VJ, Nieminen A, Coil J, Hurttia H, Larjava H (1996). Oral fluid elastase as an indicator of periodontal health. J Clin Periodontol. 23: 30-37. 
Uitto VJ, Overall CM, McCulloch C (2003). Proteolytic host cell enzymes in gingival crevice fluid. Periodontol 2000. 31: 77-104.

Weidlich P, Lopes de Souza MA, Oppermann RV (2001). Evaluation of the dentogingival area during early plaque formation. J Periodontol. 72: 901-910. 
Table 1. Periodontal indeces and demographic data of patient groups. Medians (IQR) indicated for each parameter.

\begin{tabular}{|c|c|c|c|c|c|}
\hline & $\begin{array}{c}\begin{array}{c}\text { Group } 1 \\
\text { Periodontally }\end{array} \\
\frac{\text { healthy }}{\mathrm{n}=36}\end{array}$ & $\begin{array}{c}\begin{array}{c}\text { Group } 2 \\
\text { Mild }\end{array} \\
\frac{\text { inflammatory burden }}{\mathrm{n}=21}\end{array}$ & $\begin{array}{c}\begin{array}{c}\text { Group } 3 \\
\text { Moderate }\end{array} \\
\frac{\text { inflammatory burden }}{n=104}\end{array}$ & $\begin{array}{c}\text { Group } 4 \\
\underline{\text { Strong }} \\
\frac{\text { inflammatory burden }}{\mathrm{n}=53}\end{array}$ & $\mathrm{p}$-value \\
\hline Teeth (n) & $25(22-27)$ & $26(22-28)$ & $26(23-28)$ & $25(18-27)$ & 0.094 \\
\hline Age (years) & $57(52-64)$ & $55(50-57)$ & $55(52-61)$ & $56(51-63)$ & 0.507 \\
\hline $\mathrm{BOP}(\%)$ & $4.2(1.8-5.9)$ & $13.7(12.0-16.0)$ & $6.9(4.8-13.9)$ & $19.7(14.5-28.2)$ & $<0.001$ \\
\hline PIBI & 0 & 0 & $2.5(2-5)$ & $15(8-26)$ & $<0.001$ \\
\hline PIBI x BOP (\%) & 0 & 0 & $21.0(10.9-46.1)$ & $263.9(166.6-487.2)$ & $<0.001$ \\
\hline
\end{tabular}


| Table 2. Association between periodontal indeces and consensus risk factors. Medians (IQR) indicated for each parameter.

Deleted: $x$

\begin{tabular}{|l|l|l|l|l|}
\hline & & \multicolumn{1}{|c|}{ BOP\% } & \multicolumn{1}{|c|}{ PIBI } & \multicolumn{1}{c|}{ PIBI x BOP\% } \\
\hline Gender & Males $\mathrm{n}=68$ & $11.6(6.0-17.3)$ & $5(1-14)$ & $40(9-192)$ \\
p-value & Females $\mathrm{n}=146$ & $\begin{array}{l}9.0(4.9-15.9) \\
2(0-6)\end{array}$ & $\begin{array}{l}15(0-70) \\
\mathbf{0 . 0 0 1}\end{array}$ & $\mathbf{0 . 0 0 2}$ \\
\hline Smoking & 0 (no smoking) & $8.3(4.5-15.3)$ & $2(0-5)$ & $14(0-55)$ \\
(Cigarettes & $1-10$ & $11.8(7.0-19.3)$ & $4(1-10)$ & $40(5-203)$ \\
daily) & $11-20$ & $14.5(6.9-20.0)$ & $9(5-21)$ & $112(59-321)$ \\
p-value & $>20$ & $13.7(9.7-47.6)$ & $63(32-102)$ & $927(317-5375)$ \\
\hline Diabetes & No & $\mathbf{0 . 0 3}$ & $<\mathbf{0 . 0 0 1}$ & $<\mathbf{0 . 0 0 1}$ \\
& Yes & $10.5(5.3-17.3)$ & $2(1-8)$ & $23(0-108)$ \\
p-value & $6.1(4.2-10.1)$ & $2(0-4)$ & $10(0-25)$ \\
\hline
\end{tabular}


1

2

3

4

5

6

7

8

9

10

11

12

13

14

15

Table 3. MMP-8 and TIMP-1 levels among patient groups. Medians (IQR) indicated for each parameter. $\mathrm{dA}=$ dentoAnalyzer, $\mathrm{AE}=$ Amersham ELISA.

\begin{tabular}{|c|c|c|c|c|c|}
\hline & $\frac{\begin{array}{c}\text { Group 1_ } \\
\text { Periodontally }\end{array}}{\frac{\text { healthy }}{\mathrm{n}=36}}$ & 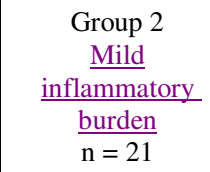 & $\begin{array}{c}\begin{array}{c}\text { Group } 3 \\
\text { Moderate }\end{array} \\
\frac{\begin{array}{c}\text { inflammatory } \\
\text { burden }\end{array}}{\mathrm{n}=104}\end{array}$ & $\begin{array}{c}\begin{array}{c}\text { Group } 4 \\
\text { Strong } \\
\text { inflammatory }\end{array} \\
\frac{\text { burden }}{\mathrm{n}=53}\end{array}$ & $\begin{array}{c}\mathrm{p}- \\
\text { values }\end{array}$ \\
\hline MMP-8 dA & $39(0-100)$ & $44(23-276)$ & $68(15-158)$ & $131(22-462)$ & 0.018 \\
\hline $\begin{array}{l}\text { MMP- } 8 \mathrm{dA} / \text { number of } \\
\text { teeth }\end{array}$ & $1.6(0-3.9)$ & $1.7(0.8-9.9)$ & $2.8(0.6-7.0)$ & $5.8(0.9-22)$ & 0.007 \\
\hline MMP-8 IFMA & $398(103-797)$ & $376(149-591)$ & $341(135-839)$ & $628(245-1131)$ & 0.112 \\
\hline $\begin{array}{l}\text { MMP-8 IFMA/number of } \\
\text { teeth }\end{array}$ & $16.4(4.2-36.3)$ & $13.2(5.2-28.7)$ & $13.7(5.3-32.7)$ & $30.9(12-54.3)$ & 0.032 \\
\hline MMP-8 AE & $35.7(14.4-82.9)$ & $32.5(19.8-69.3)$ & $39.2(17.9-80)$ & $28.8(15.1-62)$ & 0.576 \\
\hline $\begin{array}{l}\text { MMP- } 8 \mathrm{AE} / \text { number of } \\
\text { teeth }\end{array}$ & $1.55(0.77-3.47)$ & $1.31(0.8-2.48)$ & $1.49(0.75-3.38)$ & $1.31(0.68-2.83)$ & 0.921 \\
\hline dA MMP-8/TIMP-1 & $0.09(0-0.37)$ & $0.19(0.03-0.97)$ & $0.2(0.03-0.59)$ & $0.32(0.04-2.61)$ & 0.033 \\
\hline IFMA MMP-8/TIMP-1 & $1.2(0.3-2.2)$ & $0.9(0.2-3.0)$ & $1.1(0.4-2.5)$ & $1.6(0.2-6.7)$ & 0.298 \\
\hline AE MMP-8/TIMP-1 & $0.09(0.04-0.21)$ & $0.1(0.04-0.21)$ & $0.11(0.05-0.21)$ & $0.11(0.06-0.23)$ & 0.935 \\
\hline TIMP-1 & $172(111-218)$ & $170(73-227)$ & $147(95.6-229)$ & $143(76.9-258)$ & 0.770 \\
\hline
\end{tabular}


Table 4. Percentages of subjects (\%) representing peak levels of MMP-8 measured by dentoAnalyzer (dA) and IFMA adjusted to numbers of teeth.

\begin{tabular}{|c|c|c|c|c|c|c|}
\hline & $\begin{array}{l}\text { Peak } \\
\text { threshold }\end{array}$ & $\begin{array}{c}\begin{array}{c}\text { Group } 1 \\
\text { Periodontally }\end{array} \\
\frac{\text { healthy }}{\mathrm{n}=36}\end{array}$ & $\begin{array}{c}\begin{array}{c}\text { Group } 2 \\
\text { Mild }\end{array} \\
\frac{\text { inflammatory }}{\frac{\text { burden }}{n=21}}\end{array}$ & $\begin{array}{c}\begin{array}{c}\text { Group } 3 \\
\text { Moderate }\end{array} \\
\frac{\begin{array}{c}\text { inflammatory } \\
\text { burden }\end{array}}{\mathrm{n}=104}\end{array}$ & $\frac{\begin{array}{c}\text { Group } 4 \\
\text { Strong } \\
\text { inflammatory }\end{array}}{\frac{\text { burden }}{\mathrm{n}=53}}$ & $\begin{array}{l}* \mathrm{p}- \\
\text { value }\end{array}$ \\
\hline IFMA MMP-8/number of teeth & $\geq 80 \mathrm{ng} / \mathrm{ml}$ & $\begin{array}{c}-8.3 \% \\
(\mathrm{n}=3)\end{array}$ & $\begin{array}{c}-9.5 \% \\
(n=2)\end{array}$ & $\begin{array}{c}-3.8 \% \\
(n=4)\end{array}$ & $\begin{array}{c}-15.1 \%- \\
(\mathrm{n}=8)\end{array}$ & 0.104 \\
\hline dA MMP-8/number of teeth & $>15 \mathrm{ng} / \mathrm{ml}$ & $\begin{array}{l}13.9 \% \\
(n=5)\end{array}$ & $\begin{array}{l}19.0 \% \\
(\mathrm{n}=4)\end{array}$ & $\begin{array}{c}13.5 \% \\
(n=14)\end{array}$ & $\begin{array}{c}34.0 \% \\
(\mathrm{n}=18)\end{array}$ & 0.016 \\
\hline
\end{tabular}

*Contingency coefficient and Cramer V test was used for the statistical analyses. 
Figure texts

Figure 1. Boxplots representing the distributions of MMP-8 levels detected by dentoAnalyzer and IFMA adjusted to the number of teeth in study groups $(\mathrm{p}=0.007)$. Threshold line for peak dentoAnalyzer and IFMA MMP-8 levels is drawn at $15 \mathrm{ng} / \mathrm{ml}$ and $80 \mathrm{ng} / \mathrm{ml}$ levels, respectively.

Figure 2. TIMP-1 values grouped according to age of studied subjects $(\mathrm{p}<0.001)$.

Figure 3. Association between the detection of elastase activity and dentoAnalyzer MMP-8/number of teeth levels in study groups. Green represents the category of positive elastase activity and blue negative elastase activity, respectively. dentoAnalyzer MMP-8 levels v/s elastase activity in subject groups 1-4 with healthy peridontium, and with mild, moderate and strong periodontal inflammatory burden, respectively, $\mathrm{p}<0.05$;. dentoAnalyzer MMP-8 levels v/s all study groups 1-4, in subjects with positive elastase activity, $\mathrm{p}=0.002$. There were no statistically significant differences between study groups with negative elastase activities.

Deleted: subject

Figure 4. ROC (receiver operating characteristic) curve analysis for the evaluation of diagnostic sensitivity and specificity of dentoAnalyzer MMP-8/number of teeth levels in different levels of periodontal inflammatory burden (study groups 1-4 with healthy peridontium, mild, moderate and strong periodontal inflammatory burden, respectively). The area under the ROC curve, 95\% confidence interval, and p-values are given next to the figure. 


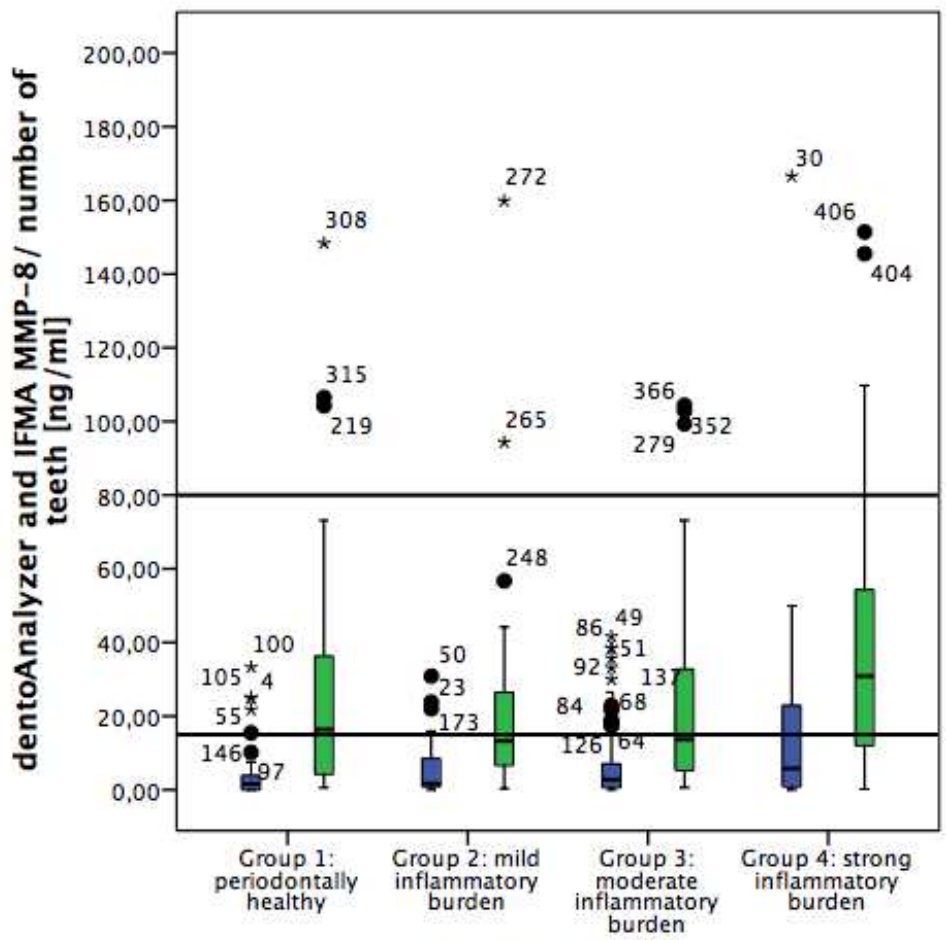

dentoAnalyzer

$\square$ IFMA

Boxplots representing the distributions of MMP-8 levels detected by dentoAnalyzer and IFMA adjusted to the number of teeth in study groups $(p=0.007)$. Threshold line for peak dentoAnalyzer and IFMA MMP-8 levels is drawn at $15 \mathrm{ng} / \mathrm{ml}$ and $80 \mathrm{ng} / \mathrm{ml}$ levels, respectively. 


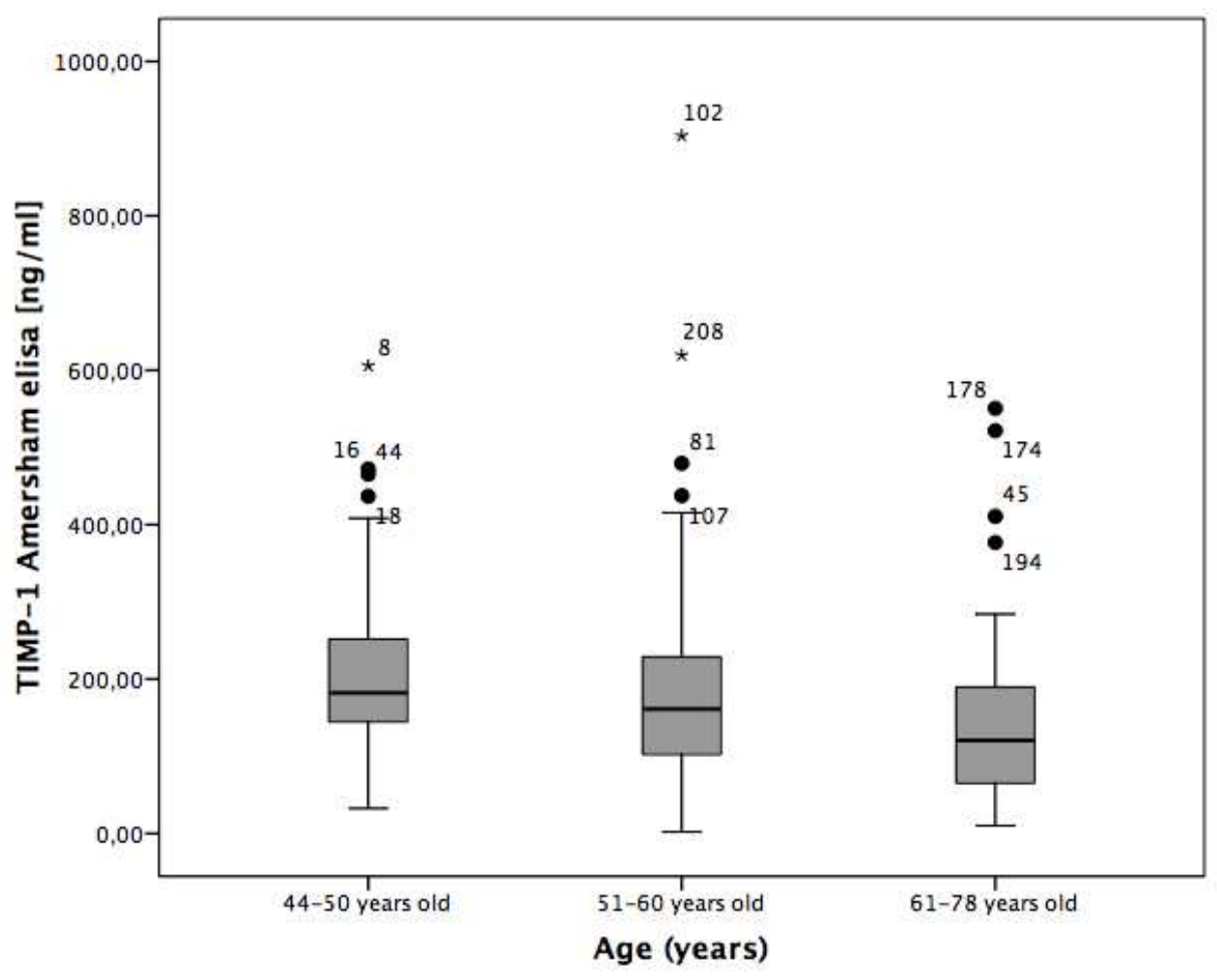

TIMP-1 values grouped according to age of studied subjects $(p<0.001)$. $166 \times 133 \mathrm{~mm}(96 \times 96 \mathrm{DPI})$ 
Association between the detection of elastase activity and dentoAnalyzer MMP-8/number of teeth levels in study groups. Green represents the category of positive elastase activity and blue negative elastase activity, respectively. dentoAnalyzer MMP-8 levels v/s elastase activity in subject groups 14 with healthy peridontium, and with mild, moderate and strong periodontal inflammatory burden, respectively, $\mathrm{p}<0.05$; dentoAnalyzer MMP-8 levels v/s all study groups 1-4, in subjects with positive elastase activity, $p=0.002$. There were no statistically significant differences between study groups having with negative elastase activities. $166 \times 133 \mathrm{~mm}(96 \times 96 \mathrm{DPI})$ 
- dentoAnalyzer MMP 8 /number of teeth, mild inflammatory burden (Group 2) vs. periodontally healthy (Group 1) $0.577(0.419-0,736), p=0.348 \mathrm{~ns}$

- dentoAnalyzer MMP $-8 /$ number of teeth. moderate inflammatory burden (Group 3) vs. periodontally healthy (Group 1)

$0.590(0.480-0.700), p=0.109$ ns

- dentoAnalyzer MMP-8/ number of teeth. strong intlammatory burden (Group 4) vs. periodontally healthy (Group 1) $0.701(0.590-0.812), p=0.002$

Figure 4. ROC (receiver operating characteristic) curve analysis for the evaluation of diagnostic sensitivity and specificity of dentoAnalyzer MMP-8/number of teeth levels in different levels of periodontal inflammatory burden (study groups 1-4 with healthy peridontium, mild, moderate and strong periodontal inflammatory burden, respectively). The area under the ROC curve, 95\% confidence interval, and $\mathrm{p}$-values are given next to the figure. $196 \times 131 \mathrm{~mm}(96 \times 96 \mathrm{DPI})$ 